\title{
Functional Testing in Glaucoma Diagnosis
}

\author{
Shivani Sethi $\cdot$ Clifton Fay $\cdot$ Kathryn E. Bollinger
}

Published online: 13 April 2013

(C) Springer Science+Business Media New York 2013

\begin{abstract}
The glaucomas are a group of diseases characterized by progressive optic neuropathy, often leading to partial or complete vision loss. Early recognition and careful monitoring for disease advancement are critical for prevention of vision loss. Frequently, asymptomatic defects in the patient's field of vision are key elements to diagnosis. Thus, it is important that the ophthalmologist stay informed regarding tools available for visual field assessment. This review will discuss the current functional testing modalities for glaucoma evaluation.
\end{abstract}

Keywords Standard automated perimetry $\cdot$ Swedish interactive threshold algorithm - Short wavelength automated technology $\cdot$ Selective perimetry $\cdot$ Retinal ganglion cell

\section{Introduction}

The technology of visual field testing has continuously evolved since the "island of vision surrounded by a sea of blindness" was first described in An Introduction to Clinical Perimetry by Scottish ophthalmologist Harry Traquair in the 1930s [1]. Visual field testing can be performed using a variety of methods. These range from basic screening tools such as the manual confrontation technique

S. Sethi · C. Fay · K. E. Bollinger $(\bowtie)$

Department of Ophthalmology, Georgia Regents University,

1120 15th Street, Augusta, GA 30912, USA

e-mail: kbollinger@gru.edu

S. Sethi

e-mail: ssethi@gru.edu

C. Fay

e-mail: cfay@gru.edu
[2], to more advanced, computer based methods. The ideal visual field test maximizes the following attributes: ease of administration, sensitivity (how well it can detect a defect), specificity (how well it can identify a normal visual field), and patient tolerability.

Boundaries of the normal visual field are approximately $60^{\circ}$ superior, $60^{\circ}$ nasal, $70^{\circ}$ inferior and $100^{\circ}$ temporal to fixation. At approximately $10^{\circ}-15^{\circ}$ nasal to the fovea, nerve fiber bundles collect at the optic nerve head. This area is devoid of photoreceptors and creates the physiologic blind spot [3]. Defects related to loss of retinal nerve fiber bundles are the most common visual field changes caused by glaucoma. Currently, these defects are most frequently assessed using standard automated perimetry, described in the first section of this review.

Individual retinal nerve fiber bundles include three general subsets of retinal ganglion cells (RGCs): magnocellular parasol cells, parvocellular midget cells, and koniocellular bistratified cells [4]. It is well established that RGCs from each of these subsets are damaged during glaucomatous neuropathy [3, 4]. Some studies suggest that individual subsets may be affected earlier or with greater severity than others depending on the patient $[3,5]$. These observations form the basis for function-specific, or selective perimetry: a perimetric method designed to capture subjective responses from specific sets of RGCs. The hypotheses behind this form of perimetry remain controversial in that currently there is no consistent evidence that RGCs are damaged selectively [6, 7]. Selective perimetry may instead be demonstrating visual field loss caused by RGCs with decreased amounts of overlap and redundancy within the visual pathway, such that a visual field defect cannot be masked [8 ${ }^{\bullet}$. Selective perimetry is discussed in greater detail within "Standard Automated Perimetry" section of this review. 
Testing methods that rely on subjective patient responses can result in the acquisition of unreliable outcome measurements that negatively affect test accuracy and precision. Objective measurements of visual function circumvent these problems. "Strategies to Minimize Test Time: FASTPAC and SITA" section of this review discusses glaucoma-relevant objective visual function tests.

\section{Standard Automated Perimetry}

Automated perimetry is currently the mainstay for evaluating functional damage related to glaucoma [4, 9, 10••]. Standard automated perimetry (SAP) using an achromatic (white-on-white) stimulus is the most commonly employed functional test and is the archetype to which newer technologies are often compared. The Humphrey Field Analyzer (HFA; Carl Zeiss Meditec, Dublin, CA, USA) is the most commonly used perimeter in the United States. However, several other manufacturers provide SAP systems worldwide.

Using computer-controlled projection, SAP presents a static white light stimulus of constant size against a white background and places the stimulus at various points in the visual field. The standard stimulus size is termed "size III" and has a diameter of $4 \mathrm{~mm}^{2}$; the size can be adjusted based on the patient's visual acuity [3,4]. The area of vision analyzed may be the central $10^{\circ}, 24^{\circ}$, or $30^{\circ}$. In the case of the Humphrey 24-2 algorithm, 54 individual points are tested [3]. The patient's threshold (the dimmest light intensity that has a $50 \%$ probability of being seen) is estimated at each point. To do this, the HFA presents a series of pre-calculated, incremental increases or decreases in static light stimulus intensity from subthreshold and suprathreshold levels, respectively [3,4]. Units of luminance, called apostilbs, represent the absolute intensity of the presented light. Measured light sensitivity is provided in a logarithmic unit, otherwise known as a decibel (dB) [3]. The threshold is provided in decibels ( $\mathrm{dB}$ ranging from 0 to $50 \mathrm{~dB}$, where $0 \mathrm{~dB}$ is the brightest and $50 \mathrm{~dB}$ is the most attenuated target the perimeter is able to project [11]. It is important to note that a decibel is a relative unit. It represents the same amount of change in intensity across perimeters; however, the absolute light intensity (measured in apostilbs), of a stimulus may not be the same between machines [3].

Upon test completion, a normative database compares the patient's sensitivities to those obtained from age-matched normal individuals. The HFA then provides the clinician with a printout (Fig. 1) that includes multiple scales used to interpret the visual field. At the top of the printout, patient identification and testing parameters are provided. Three test reliability indices are reported on the upper left of the printout. They include: (1) the number of times the patient responds when the stimulus is presented within his/ her blind spot (fixation losses), (2) the number of times a patient responds without a stimulus (false positive rate), and (3) the number of times the patient fails to respond to a stimulus brighter than the previously estimated threshold at that test location (false negative rate). High values in each of these parameters indicate a visual field with low reliability and one that should be interpreted with caution $[3,11,12 \bullet]$.

The HFA then provides a numerical map of the patient's threshold values adjacent to a corresponding gray scale. These plots are not adjusted for age or diffuse loss and so are not reliable indicators of visual field defects [12•]. No diagnosis should be made based solely on the gray scale [11]. It may be helpful, however, in highlighting areas that need further review and may function as a gross map by which to educate the patient about his or her test results.

Within the HFA-provided print-out, the total deviation plot is located below the gray scale. It provides the clinician with a representation of age-corrected deviation from normal. In this analysis, each location tested is graded as normal or abnormal via a $p$ value $[4,11,12 \bullet]$. The total deviation plot does not correct for generalized depression of sensitivity that can be caused by cataract or pupil size. To do this, the pattern deviation plot is derived from the total deviation plot. It is located to the right of the total deviation plot. The pattern deviation plot represents localized change and provides p-values in addition to agecorrected normal values. Because it is a sensitive tool for identifying localized defects, the pattern deviation plot is a very useful plot for glaucoma detection [4].

Automated analyses also generate global indices that provide the ophthalmologist with mathematical evaluations of the visual field. These indices are found on the bottom right of the printout. The mean deviation (MD) index reflects diffuse change and is a weighted average of all the points in the total deviation plot [3]. The more negative the MD value, the brighter the average stimulus needed for patient response [12•]. The pattern standard deviation (PSD) index reflects variability across the visual field [13]. A higher PSD value reflects the presence of a more focal visual field defect [12•].

The glaucoma hemifield test (GHT) is another HFAprovided automated evaluation. GHT analyzes differences in threshold sensitivity between the upper and lower hemifields as a method to separate normal from glaucomatous visual fields. The test is based on observations that glaucomatous visual field defects typically show asymmetry with respect to the horizontal midline. The test algorithm compares five corresponding sector pairs based on the normal anatomy of the retinal nerve fiber layer [14], and analyzes the difference between the upper and lower hemifields. When used in correlation with the pattern 
Fig. 1 Humphrey visual field 24-2 right eye
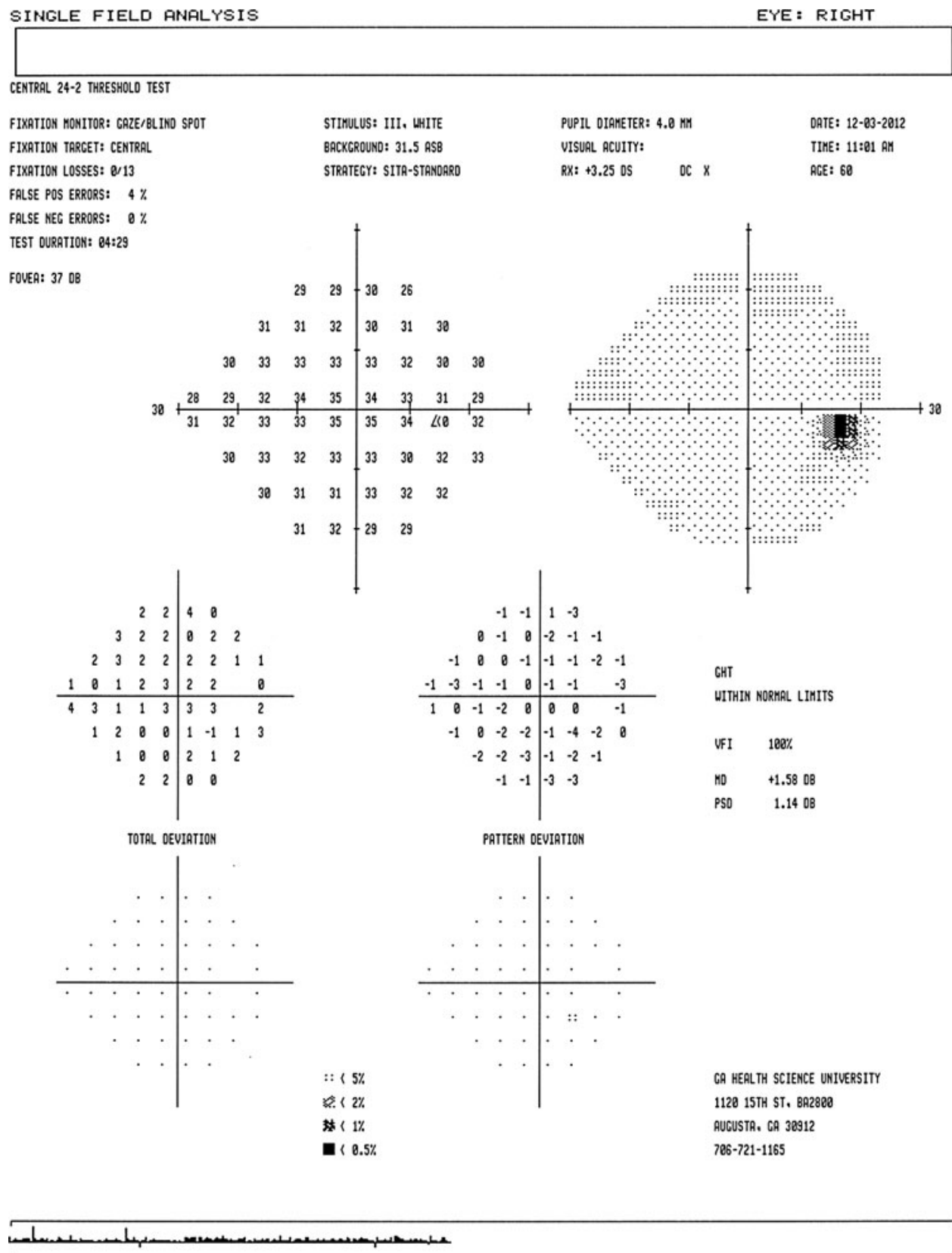

E 2010 CARL ZEISS MEOITEC HFA II $758-13988-5.1 .1 / 5.1 .1$

deviation plot, the GHT has been shown to have high sensitivity and specificity for changes associated with early glaucoma [15].

Several confounding variables affect SAP results. The technician must instruct patients on how to perform the exam, properly correct any relevant refractive error, and hold a supervisory role throughout the test, redirecting patients if necessary. A recent study showed that independent of glaucoma severity stage, an inexperienced perimetrist could negatively influence the test result, possibly lowering the mean deviation [16•].
Accurate results are also patient-dependent. Alertness, cooperation and fixation all contribute to an effective test. Such factors are negatively influenced by lengthy test times and inconsistent stimulus visualization ability, leading to unreliable results. The Ocular Hypertension Study revealed that three consecutive and reliable visual field test results have greater specificity and stability than either one or two consecutive tests to confirm a visual field abnormality $[17 \bullet \cdot]$. In addition, other patient-related physiologic factors contribute to decreased test reliability. Increasing age is associated with reduced retinal threshold sensitivity [18], 
and the normally aging lens may cause enough light scatter to influence threshold measurements [19]. These confounding variables, however, are largely corrected by the HFA's normative database and via the total and pattern deviation plots.

Even performed reliably, SAP has inherent critical limitations. In many eyes, the temporal relationship between structural and functional defects can be delayed. [20, 21]. Therefore, SAP does not provide ideal sensitivity for detection of early glaucomatous changes because a certain number of RGCs must be lost (25-50 \%) before a visual field defect is detected [22, 23, 24].

\section{Strategies to Minimize Test Time: FASTPAC and SITA}

Full threshold testing can be tiresome for the patient, leading to exacerbation of patient-dependant limitations including alertness and cooperation. To minimize test time, two alternative threshold strategies have been created: FASTPAC and the Swedish interactive threshold algorithm (SITA). In contrast to full threshold SAP, FASTPAC changes threshold stimulus intensity in larger increments and crosses the threshold only once [3]. Test time is significantly reduced, but this method can deliver short-term fluctuations and has been reported to underestimate defect severity [25, 26]. The FASTPAC strategy has become largely obsolete since the application of SITA [27].

The SITA strategy has two commercially available versions: SITA standard and SITA fast. When measured against full threshold SAP, SITA standard takes about half the time to complete, and SITA fast is even shorter in duration [3]. To accomplish this, the SITA strategy not only utilizes available knowledge from normal and glaucomatous visual fields to estimate the threshold value $[27,28]$, the algorithm is constantly being updated throughout the test based on patient response [3]. Through this approach, SITA standard is able to decrease the actual number of stimuli presented to the patient compared to full threshold SAP, making test time approximately $7 \mathrm{~min}$ per eye [4]. SITA fast allows for even shorter examinations by using larger jumps in stimulus intensity between test spots, specifically in $4 \mathrm{~dB}$ steps that reverse once as opposed to the standard 4-2 $\mathrm{dB}$ increments, that cross threshold twice [4]. SITA fast may be the test of choice in patients who are unable to tolerate a longer exam.

In a study by Budenz et al., when evaluating the detection of mild glaucomatous field loss compared to full threshold SAP, SITA standard had a sensitivity of $92 \%$ and SITA fast had a sensitivity of $85 \%$. Both SITA standard and SITA fast showed $100 \%$ sensitivity for severe visual field loss [27]. In addition, studies have found less test/retest variability with SITA standard when compared to full threshold SAP [28]. Variability is comparable with SITA fast, but only when based on threshold sensitivities of more than $25 \mathrm{~dB}$ [29]. Reliable sensitivity and specificity, along with decreased variability, have lead to widespread use of SITA algorithms in the clinical setting.

\section{Selective Perimetry: SWAP, FDT, and HPRP}

A significant number of RGCs must be depleted before a visual field defect is measureable using SAP [30]. Selective perimetry was developed to identify patients with visual field loss not detected by SAP. This visual field testing method stimulates responses from specific subsets of ganglion cells, those derived from magnocellular, parvocellular, or koniocellular pathways. Compared to SAP, selective perimetry methods target only a small fraction of the visual system. Because fewer cells are tested, there is reduced overlap between RGC receptive fields. Selective perimetry's ability to examine patient responses that correlate to a less redundant aspect of visual function may account for earlier detection of glaucomatous vision defects with this technology as compared to SAP [8•]. The following section will describe the current methods available to selectively measure a visual field based on isolated cell types.

\section{Short Wavelength Automated Perimetry}

Short wavelength automated perimetry (SWAP) tests the function of an RGC subgroup in the koniocellular pathway: the blue-yellow small bistratified ganglion cells [31]. This is done using a large blue stimulus (size V, $64 \mathrm{~mm}^{2}$ ) against a yellow background. It is available on HFA II (Carl Zeiss Ophthalmic Systems, Humphrey Division, Dublin, CA, USA) and on the Octopus 300 and 900 perimeters. Both full threshold and SITA strategies can be used, and aside from the larger stimulus size, all features of testing are similar to SAP.

SWAP has shown improved sensitivity for identification of early glaucomatous vision loss compared with SAP [32]. In fact, SWAP may detect functional deficits up to 5 years earlier than SAP in some patients [32, 33]. In addition, in many cases, defects detected by SWAP correlate with early structural glaucomatous damage [34, 35]. Moreover, some longitudinal studies suggest that SWAP may provide a better predictor of photographically-determined structural change than SAP $[36,37 \bullet, 38]$.

A disadvantage of the SWAP technique is longer test time; this can decrease both patient satisfaction and clinical practice efficiency. Integration of the SITA threshold strategy has reduced test duration [39]. However, SWAP is also influenced by media opacities and shows significant intratest and intertest variability compared with SAP. This 
may lead to false positive test results in many glaucoma suspects [40-42].

\section{Frequency Doubling Technology}

Another available form of selective perimetry is frequencydoubling technology (FDT). FDT stimulates large-diameter ganglion cells in the magnocellular pathway, specifically a subset of parasol cells called $M_{\mathrm{y}}$ ganglion cells [43]. The test employs the "frequency doubling illusion" as described by Kelly in 1966. The "illusion" consists of alternating dark and light bars shown at high temporal frequency and low spatial frequency. When viewed, the bars appear to double in number [44]. Specifically, the FDT stimulus is a vertical sine wave grating with spatial frequency of 0.25 c/deg displayed with counterphase flickering at a temporal frequency of $25 \mathrm{~Hz}$. The C-20 presentation pattern tests the central $20^{\circ}$ of the visual field and presents a $10^{\circ}$ square target at 16 locations (four per quadrant). A small central target $\left(5^{\circ}\right)$ is also projected on the macular region. For the $\mathrm{N}-30$ presentation, two additional points (total of 19 points) are presented within the nasal visual field.

Both screening and full threshold algorithms are available for FDT, and the test time is short: approximately 1 and $5 \mathrm{~min}$, respectively [10••]. The Humphrey FDT Perimeter (Carl Zeiss Meditec; Dublin, CA, USA) is easy to transport and relatively inexpensive. In addition, the test results are less affected by media opacities than SWAP [45]. Studies have shown FDT to have a high sensitivity and specificity for differentiating non-glaucoma individuals from those with glaucoma [46]. In fact, evidence also suggests that some false positive results may actually represent detection of glaucomatous damage earlier than SAP [47••]. However, in general, when used in the clinic setting, the FDT screening protocol offers levels of specificity and sensitivity similar to SAP. FDT has also been evaluated as a population-based screening tool [48-50]. At the population level, it does not appear to have sensitivity and specificity measures needed to detect early glaucoma. However, it may be useful for population-based testing when the goal is detection of advanced disease.

The Matrix perimeter (Carl Zeiss Meditec; Dublin, CA. Welch Allyn) is the most recent FDT testing system. It provides video eye monitoring, along with improved spatial resolution of visual field defects. This is done by using a greater number of testing locations and smaller, $4^{\circ}$ square stimuli, spaced in a $6^{\circ}$ grid. The testing pattern is similar to the 24-2 protocol used in the HFA. In addition, the Matrix perimeter offers programs containing $2^{\circ}$ wide stimuli for testing the central visual field (10-2), improved threshold calculations, and video eye monitoring [51, 52]. Like FDT, Matrix FDT is able to offer a sensitivity and specificity similar to SAP [53]; it does, however, have longer test times than standard FDT (6 min per eye).

High-Pass Resolution Perimetry

High-pass resolution perimetry (commercially available as the Ophthimus High Pass Resolution Perimeter, Hightech Vision, Goteburg, Sweden) allows another selective evaluation of visual field function that tests the parvocellular system of RGCs. Here, ring shaped stimuli of different sizes but with fixed contrast are presented at 50 different locations in the central $30^{\circ}$ of the visual field $[54,55]$. Some evidence suggests that HPRP may be able to diagnose early glaucomatous damage [56, 57]. For example, Iester et al. compared SAP (HFA 30-2), FDT (C-20), and HPRP parameters in 52 eyes determined to have glaucoma by disc examination, visual field, or both and in 30 eyes with IOP of more than $21 \mathrm{mmHg}$ but no other abnormalities. Within the entire group, a significant correlation was found between HFA indices and those of HPRP or FDT. However, FDT detected 29 visual fields as abnormal despite normal HFA and HPRP detected 26 fields as abnormal despite normal HFA. The authors concluded that FDT and HPRP may be useful to identify early glaucomatous disease [57].

Other studies have not found that HPRP is superior to other perimetry tests. Sample et al. compared HPRP with SWAP, FDT N-30, and SAP. At a specificity of $90 \%$ for photographic progression of the optic disc, the sensitivity of the best SWAP parameter was $45 \%$, that for FDT was $68 \%$, and that for HPRP was $52 \%$ [58].

Electrophysiological studies provide a novel avenue for diagnosis of glaucomatous nerve damage. These techniques circumvent the inherent subjectivity that can be problematic to the visual function tests described above. Many of the following methods are well studied in retinal disease and hold promise for analysis of optic nerve disorders including glaucoma.

\section{Electroretinography}

Electroretinography (ERG) provides a record of the bioelectrical changes that occur across the retina. This activity is recorded using an electrode placed on the corneal or bulbar conjunctival surface. The classic ERG displays a negative a-wave (representing the photoreceptors) and a positive b-wave (representing the bipolar cell layer). These recorded electrical changes occur in response to a visual stimulus such as a flash of light. Comparison is then made to characteristic normal responses [59, 60]. Evaluation of electrophysiological techniques using experimental glaucoma models suggests that they may be helpful in detection of early glaucomatous changes [61]. 
The prototypal ERG provides the clinician with a summated, pan-retinal response, making localization of retinal pathology difficult. However, in the early 1990s, a multifocal stimulus was first used to evaluate topographic retinal responses [62]. The multifocal ERG (mfERG) has since come into widespread clinical use. It offers insight into many vision-threatening diseases that affect the outer retina including retinitis pigmentosa, macular degeneration, and retinal detachment.

With respect to glaucoma diagnosis, no simple correlation exists between mfERG responses and the retinal dysfunction observed in glaucomatous visual field defects. This is because the contribution of retinal ganglion cell activity to the mfERG response is indirect and complicated to measure [63]. However, several different mfERG stimulus and analysis paradigms have been evaluated for use in glaucoma testing [64]. In fact, some recent studies show good differentiation between normal and glaucomatous patients [65•]. In addition, recent work using a laserinduced primate model of glaucoma showed significant correlations between focal measurements of global flash mfERG, regional retinal nerve fiber layer thickness, and local visual field sensitivity [66].

Despite promising initial studies, early detection of glaucoma using mfERG has not yet been clinically established [10••, 67]. In fact, some authors have suggested that mfERG changes do not show a clinically useful correlation with glaucomatous damage [68]. However, mfERG technology continues to evolve and there is hope that it will become an important tool for diagnosis and management of glaucoma [68-70].

The pattern electroretinogram (PERG) can detect RGC dysfunction in glaucoma and glaucoma suspects [71••, 72]. PERG presents a reversing black and white gradient, or checkerboard stimulus, to the central retina and provides a direct measure of RGC function [71••, 72, 73]. Preliminary data suggest that PERG abnormalities are measureable in early glaucoma and show moderate correlation with SAP values and with structural optic nerve assessments [74-76]. In addition, recent studies indicate that reversal of PERG abnormalities occurs following medical and surgical intervention for glaucoma. Therefore, PERG may offer a useful measure both of RGC dysfunction in early-stage disease (preperimetric glaucoma) and of an individual patient's response to treatment $[72,77]$. Of note, PERG is negatively correlated with intraocular pressure, age, media opacity, and visual acuity [71••]. Therefore, to be clinically useful, results must be compared to a normative database and adjustments made for these factors. [71••, 78] Also, PERG requires a more skilled examiner than that for automated visual field tests [79]. A simplified PERG known as PERGLA (Pattern Electroretinogram for
Glaucoma Detection; Glaid, Lace Elettronica, Pisa, Italy) rectifies some of the shortcomings of standard PERG. This updated paradigm is fast, completely automated, and uses skin rather than corneal electrodes. PERGLA has an improved signal-to-noise ratio compared with standard PERG, and is less affected by eye movements [80]. PERGLA amplitude is also affected by age, IOP, cataract, and visual acuity [80]. In addition, amplitudes show overlap between normal and glaucomatous individuals. [81-83]

The photopic negative response (PhNR) has also been evaluated as a method of glaucoma diagnosis. This response originates primarily from activity of RGCs and consists of a slow negative potential that follows the b-wave on ERG [84, 85]. Studies have found reduction in $\mathrm{PhNR}$ amplitude to be a sensitive indicator of glaucoma [84] and the focal PhNR to be a valid method to detect functional loss in early and intermediate glaucoma [86]. The PhNR amplitude also correlates with morphology and structural changes seen in eyes with open-angle glaucoma [87]. Advantages of the PhNR over the PERG include less media opacity effect and no refractive correction requirement [84]. However, media opacities do still have some effect on the PhNR; dense cataract or vitreous hemorrhage make focal PhNR testing impossible. Also, during testing, the patient must gaze at each fixation point for $1 \mathrm{~min}$ with minimal eye movement. Thus, this test has some of the patient reliability issues faced with subjective perimetry [88].

\section{Visual Evoked Potentials}

Visual evoked potentials (VEP) are electrical signals produced by the visual cortex in response to a light stimulus. These responses have been traditionally used to evaluate macular function, but recent work has also focused on optic nerve disease [61]. Specifically, the multifocal VEP (mfVEP) may be useful in glaucoma detection [10••]. Like the $\mathrm{mfERG}$, it provides topographic mapping of retinal responses. Multiple VEPs can be recorded rapidly and simultaneously [40]. Hood et al. [89] have found the mfVEP to be a promising alternative for patients who repeatedly record unreliable visual fields by standard perimetry. Moreover, using interocular analysis, mfVEP can detect mild, unilateral defects with high sensitivity. However, this test can miss abnormalities when damage exists in the same location within both eyes [89, 90]. Multifocal VEP and SAP have been shown to correspond in up to $81 \%$ of early glaucomatous defects [91]. In addition, in cases of uncertain or subjective field defects, studies have found mfVEP to be a valuable adjunct to SAP in finalizing the diagnosis of glaucoma [92]. 


\section{Conclusion}

There is no assessment of visual function that approaches the "ideal test." However, in comparison to full threshold SAP, algorithms such as SITA have improved many test performance measures.

Currently, the most commonly used selective perimetry modalities are FDT and SWAP. For routine clinical use, the FDT screening protocol offers levels of specificity and sensitivity comparable to SAP. In addition, it may hold promise as a tool to diagnose glaucoma in the communitybased setting. SWAP is likely capable of detecting glaucoma earlier than SAP but may indicate false positive results more frequently than SAP.

Objective methods of evaluating visual function offer potential advantages over traditional subjective visual field tests. However, further studies are needed in order to determine the role of these technologies in glaucoma diagnosis. In addition, equipment availability, provider expertise, and patient acceptance issues currently limit adoption of an objective method for routine clinical use. Characteristics and limitations differ among the modalities used for functional testing in glaucoma. Understanding how these methods vary will assist the physician in making a diagnosis of disease.

Disclosure No potential conflicts of interest relevant to this article were reported.

\section{References}

Papers of particular interest, published recently, have been highlighted as:

- Of importance

•. Of major importance

1. Grzybowski A. Harry Moss Traquair (1875-1954) Scottish ophthalmologist and perimetrist. Acta Ophthalmol. 2009;87(4): $455-9$.

2. Trobe JD, Acosta PC, Krischer JP, et al. Confrontation visual field techniques in the detection of anterior visual pathway lesions. Ann Neurol. 1981;10(1):28-34.

3. Allingham R, Damji K, Freedman S, et al. Shields' textbook of glaucoma. Philadelphia: Lippincott Williams \& Williams; 2005 471-483.

4. Shaarawy T, Sherwood M, Hitchings R, et al. Glaucoma volume 1: medical diagnosis \& therapy. Amsterdam: Elsevier; 2009.

5. Anderson RS, O'Brien C. Psychophysical evidence for a selective loss of M ganglion cells in glaucoma. Vision Res. 1997;37(8): 1079-83.

6. McKendrick AM, Badcock DR, Morgan WH. Psychophysical measurement of neural adaptation abnormalities in magnocellular and parvocellular pathways in glaucoma. Invest Ophthalmol Vis Sci. 2004;45(6): 1846-53.
7. Martin L, Wanger P, Vancea L, et al. Concordance of high-pass resolution perimetry and frequency-doubling technology perimetry results in glaucoma: no support for selective ganglion cell damage. J Glaucoma. 2003;12(1):40-4.

8. - Spry PG, Johnson CA, Mansberger SL, Cioffi GA. Psychophysical investigation of ganglion cell loss in early glaucoma. J Glaucoma. 2005;14(1):11-19. Results of this investigation suggest that either reduced redundancy and/or preferential loss of larger sized ganglion cells explains the cell loss that occurs early in the course of glaucoma. Results do not support selective loss of magnocellular or parvocellular ganglion cell subpopulations.

9. Spry, PG, Johnson CA. Advances in automated perimetry. focal points: clinical modules for ophthalmologists, module 10. San Francisco: American Academy of Ophthalmology; 2002.

10. •- Turalba AV, Grosskreutz C. A review of current technology used in evaluating visual function in glaucoma. Semin Ophthalmol. 2010;25(5-6):309-316. Review of current algorithms and functional tests that are being used in the diagnosis and management of glaucoma.

11. Thomas R, George R. Interpreting automated perimetry. Indian J Ophthalmol. 2001;49(2):125-40.

12. - Alencar LM, Medeiros FA. The role of standard automated perimetry and newer functional methods for glaucoma diagnosis and follow up. Indian J Ophthalmol. 2011;59 Suppl:S53-S58. Review of standard automated perimetry, including a review of the Humphrey visual field printout as well as the FDT 24-2 Matrix printout.

13. Choplin NT, Edwards RP. Visual field testing with the Humphrey field analyzer: a text and clinical atlas. 2nd ed. Thorofare: Slack Inc; 1999.

14. Asman P, Heijl A. Glaucoma hemifield test. Automated visual field evaluation. Arch Ophthalmol. 1992;110(6):812-9.

15. Johnson CA, Sample PA, Cioffi GA, et al. Structure and function evaluation (SAFE): I. criteria for glaucomatous visual field loss using standard automated perimetry (SAP) and short wavelength automated perimetry (SWAP). Am J Ophthalmol. 2002;134(2): $177-85$.

16. - Junoy Montolio FG, Wesselink C, Gordijn M, et al. Factors that influence standard automated perimetry test results in glaucoma: test reliability, technician experience, time of day and season. Invest Ophthalmol Vis Sci. 2012;53(11):7010-7017. Paper reviews many possible barriers to an accurate visual field examination.

17. •• Keltner JL, Johnson CA, Levine RA, et al. Normal visual field test results following glaucomatous visual field end points in the Ocular Hypertension Treatment Study. Arch Ophthalmol. 2005;123(9):1201-1206. Landmark study by the Ocular Hypertension Treatment Study group which highlights the need for consecutive visual field testing in the diagnosis of glaucoma.

18. Spry PG, Johnson CA. Senescent changes of the normal visual field: an age-old problem. Optom Vis Sci. 2001;78(6):436-41.

19. The AGIS Investigators. The advanced glaucoma intervention study, 6: effect of cataract on visual field and visual acuity. Arch Ophthalmol. 2000;118(12):1639-52.

20. Kass MA, Heurer DK, Higginbotham EJ, et al. The Ocular Hypertension Treatment Study: a randomized trial determines that topical ocular hypotensive medication delays or prevents the onset of primary open-angle glaucoma. Arch Ophthalmol. 2002;120(6):701-13.

21. Miglior S, Zeyen T, Pfeiffer N, et al. Results of the European Glaucoma Prevention Study. Ophthalmology. 2005;112(3): 366-75.

22. Kerrigan-Baumrind LA, Quigley HA, Pease ME, et al. Number of ganglion cells in glaucoma eyes compared with threshold visual 
field tests in the same person. Invest Ophthalmol Vis Sci. 2000;41(3):741-8

23. • Jampel HD, Singh K, Lin SC, Chen TC, Francis BA, Hodapp E, Samples JR, Smith SD. Assessment of visual function in glaucoma: a report by the American Academy of Ophthalmology. Ophthalmology. 2011;118(5):986-1002. Report by the American Academy of Ophthalmology that reviews the current methods of functional testing in glaucoma diagnosis as well as the published literature that surrounds these methods.

24. Zeyen TG, Caprioli J. Progression of disc and field damage in early glaucoma. Arch Ophthalmol. 1993;111(1):62-5.

25. Schaumberger M, Schafer B, Lachenmayr BJ. Glaucomatous visual fields. FASTPAC versus full threshold strategy of the Humphrey field analyzer. Invest Ophthalmol Vis Sci. 1995;36(7):1390-7.

26. O'Brien C, Poinoosawmy $\mathrm{D}$, Wu $\mathrm{J}$, et al. Evaluation of the Humphrey FASTPAC threshold program in glaucoma. $\mathrm{Br} \mathrm{J}$ Ophthalmol. 1994;78(7):516-9.

27. Budenz DL, Rhee P, Feuer WJ, et al. Sensitivity and specificity of the Swedish interactive threshold algorithm for glaucomatous visual field defects. Ophthalmology. 2002;109(6):1052-8.

28. Bengtson B, Heijl A, Olsson J. Evaluation of new threshold visual field strategy, SITA, in normal subjects. Swedish interactive threshold algorithm. Acta Ophthalmol Scand. 1998;76(2): $165-9$.

29. Artes PH, Iwas A, Ohno $\mathrm{Y}$, et al. Properties of perimetric threshold estimates from full threshold, SITA standard, and SITA fast strategies. Invest Ophthalmol Vis Sci. 2002;43(8):2654-9.

30. Kerrigan-Baumrind LA, Quigley HA, Pease ME, et al. Number of ganglion cells in glaucoma eyes compared with threshold visual field tests in the same persons. Invest Ophthalmol Vis Sci. 2000;41(3):741-8.

31. Dacey DM, Packer OS. Color coding the primate retina: diverse cell types and cone specific circuitry. Curr Opin Neurobiol. 2003;13(4):421-7.

32. Johnson CA, Adams AJ, Casson EJ, et al. Progression of early glaucomatous visual field loss as detected by blue-on yellow and standard white on white automated perimetry. Arch Ophthalmol. 1993;111(5):651-6.

33. Demirel S, Johnson CA. Incidence and prevalence of short wavelength automated perimetry deficits in ocular hypertensive patients. Am J Ophthalmol. 2001;131(6):709-15.

34. Ferreras A, Polo V, Larrosa JM, et al. Can frequency-doubling technology and short-wavelength automated perimetries detect visual field defects before standard automated perimetry in patients with preperimetric glaucoma? J Glaucoma. 2007;16(4): 372-83.

35. Johnson CA, Adams AJ, Casson EJ, et al. Blue-on-yellow perimetry can predict the development of glaucomatous visual field loss. Arch Ophthalmol. 1993;111(5):645-50.

36. Girkin CA, Emdadi A, Sample PA, et al. Short-wavelength automated perimetry and standard perimetry in the detection of progressive optic disc cupping. Arch Ophthalmol. 2000;118(9): 1231-6.

37. - Polo V, Larrosa JM, Pinilla I, et al. Predictive value of shortwavelength automated perimetry: a 3-year follow-up study. Ophthalmology. 2002;109(4):761-765. The authors performed SWAP 30-2 and red-free RNFL photography in 160 eyes of 83 patients and followed up the patients with 30-2 SAP for at least 3 years. Of the 58 eyes with abnormal SWAP results, SAP defects developed in $19 \%$, as opposed to only $4 \%$ of the 102 eyes with normal baseline SWAP.

38. Bayer AU, Maag KP, Erb C. Detection of optic neuropathy in glaucomatous eyes with normal standard visual fields using a test battery of short-wavelength automated perimetry and pattern electroretinography. Ophthalmology. 2002;109(7):1350-61.
39. Delgado MF, Nguyen NT, Cox TA, et al. Automated perimetry: a report by the American Academy of Ophthalmology. Ophthalmology. 2002;109(12):2362-74.

40. Blumenthal EZ, Sample PA, Zangwill L, et al. Comparison of long-term variability for standard and short-wavelength automated perimetry in stable glaucoma patients. Am J Ophthalmol. 2000;129(3):309-13.

41. Hutchings N, Hosking SL, Wild JM, et al. Long-term fluctuations in short-wavelength automated perimetry in glaucoma suspects and glaucoma patients. Invest Ophthalmol Vis Sci. 2001;42(10):2332-7.

42. Blumenthal EZ, Sample PA, Berry CC, et al. Evaluating several sources of variability for standard and SWAP visual fields in the glaucoma patients, suspects and normal. Ophthalmology. 2003;110(10):1895-902.

43. Johnson CA, Samuels SJ. Screening for glaucomatous visual field loss with frequency-doubling perimetry. Invest Ophthalmol Vis Sci. 1997;38(2):413-25.

44. Kelly DH. Frequency doubling in visual responses. J Opt Soc Am. 1966;56:1628-33.

45. Anderson AJ, Johnson CA. Frequency doubling technology perimetry. Ophthalmol Clin North Am. 2003;16(2):213-25.

46. Iester M, Mermoud A, Schnyder C. Frequency doubling technique in patients with ocular hypertension and glaucoma: correlation with Octopus perimeter indices. Ophthalmology. 2000;107(2):288-94.

47. • Wadood AC, Azuara-Blanco A, Aspinall P, et al. Sensitivity and specificity of frequency-doubling technology, tendencyoriented perimetry, and Humphrey Swedish interactive threshold algorithm-fast perimetry in a glaucoma practice. Am J Ophthalmol. 2002;133(3):327-32. A prospective study evaluating the sensitivity and specificity of the screening mode of the Humphrey frequency-doubling technology (FDT), Octopus tendency-oriented perimetry (TOP), and the Humphrey Swedish Interactive Threshold Algorithm (SITA)-fast (HSF) in patients with glaucoma. The AUC for at least 1 abnormal FDT test location was 0.93, with a sensitivity of $91 \%$ and a specificity of $96 \%$.

48. Yamada N, Chen PP, Mills RP, et al. Screening for glaucoma with frequency-doubling technology and Damato campimetry. Arch Ophthalmol. 1999;117(11):1479-84.

49. Tatemichi M, Nakano T, Tanaka K, et al. Performance of glaucoma mass screening with only a visual field test using frequency-doubling technology perimetry. Am J Ophthalmol. 2002;134(4):529-37.

50. Wang $\mathrm{YX}, \mathrm{Xu} \mathrm{L}$, Zhang $\mathrm{RX}$, et al. Frequency-doubling threshold perimetry in predicting glaucoma in a populationbased study: the Beijing Eye Study. Arch Ophthalmol. 2007;125(10):1402-6.

51. Lamparter J, Schulze A, Hoffmann EM. Frequency-doubling technology: a new method for determining glaucomatous visual field defects. Ophthalmology. 2009;106(8):709-13.

52. Johnson CA, Cioffi GA, Van Buskirk EM. Frequency doubling technology perimetry using a 24-2 stimulus presentation pattern. Optom Vis Sci. 1999;76(8):571-81.

53. Spry PG, Hussin HM, Sparrow JM. Clinical evaluation of frequency doubling technology perimetry using the Humphrey matrix 24-2 threshold strategy. Br J Ophthalmol. 2005;89(8): 1031-5.

54. Frisen L. High pass resolution perimetry: a clinical review. Doc Ophthalmol. 1993;83(1):1-25.

55. Frisen L. High pass resolution perimetry: central-field neuroretinal correlates. Vision Res. 1995;35(2):293-301.

56. Chauhan BC, House PH, McCormick TA, et al. Comparison of conventional and high pass resolution perimetry in a prospective study of patients with glaucoma and healthy controls. Arch Ophthalmol. 1999;117(1):24-33. 
57. Iester M, Altieri M, Vittone $\mathrm{P}$, et al. Detection of glaucomatous visual field defect by nonconventional perimetry. Am J Ophthalmol. 2003;135(1):35-9.

58. Sample PA, Medeiros FA, Racette L, et al. Identifying glaucomatous vision loss with visual-function-specific perimetry in the diagnostic innovations in glaucoma study. Invest Ophthalmol Vis Sci. 2006;47(8):3381-9.

59. Chan HL, Brown B. Multifocal ERG changes in glaucoma. Ophthalmic Physiol Opt. 1999;19(4):306-16.

60. Basic and clinical science course (BCSC) Section 10: glaucoma. San Francisco: American Academy of Ophthalmology; 20102011.

61. Harwerth RS, Crawford ML, Frishman LJ, et al. Visual field defects and neural losses from experimental glaucoma. Prog Retin Eye Res. 2002;21(1):91-125.

62. Sutter EE, Tran D. The field topography of ERG components in man-I. The photopic luminance response. Vision Res. 1992;32(3):433-46.

63. Kretschmann U, Bock M, Gockeln R, et al. Clinical applications of multifocal electroretinography. Doc Ophthalmol. 2000; 100(2-3):99-113.

64. Lai TY, Chan M, Lai RY, et al. The clinical applications of multifocal electroretinography: a systematic review. Surv Ophthalmol. 2007;52(1):61-96.

65. - Chu PH, Chan HH, Brown B. Luminance modulated adaptation of global flash mrERG: fellow eye losses in asymmetric glaucoma. Invest Ophthalmol Vis Sci. 2007;48:2626-2633. Chu et al evaluated mfERG testing in glaucoma subjects with abnormal SAP in 1 eye and normal SAP in the other eye. They also followed normal subjects. The amplitude of response was reduced in the better eye of subjects with glaucoma compared with those without glaucoma.

66. Luo X, Patel NB, Harwerth RS, Frishman LJ. Loss of the lowfrequency component of the global-flash multifocal electroretinogram in primate eyes with experimental glaucoma. Invest Ophthalmol Vis Sci. 2011;52(6):3792-804.

67. Chan HH, Brown B. Pilot study of the multifocal electroretinogram in ocular hypertension. Br J Ophthalmol. 2000;84(10): 1147-53.

68. Sakemi F, Yoshii M, Okisaka S. Multifocal electroretinograms in early primary open angle glaucoma. Jpn J Ophthalmol. 2002;46(4):443-50.

69. Miguel-Jiménez JM, Boquete L, Ortega S, et al. Glaucoma detection by wavelet-based analysis of the global flash multifocal electroretinogram. Med Eng Phys. 2010;32(6):617-22.

70. Miguel-Jiménez JM, Ortega S, Boquete L, et al. Multifocal ERG wavelet packet decomposition applied to glaucoma diagnosis. Biomed Eng Online. 2011;17(10):37.

71. •• Porciatti V, Ventura LM. Normative data for a user-friendly paradigm for pattern electroretinogram recording. Ophthalmology. 2004;111(1):161-8. This paper describes a paradigm for recording PERG in a fast and automated way. Normative data are provided for a population of 93 healthy individuals of different age.

72. Ventura LM, Porciatti V, Ishida K, et al. Pattern electroretinogram abnormality and glaucoma. Ophthalmology. 2005;112(1): $10-9$.

73. Holder GE. Pattern electroretinography (PERG) and an integrated approach to visual pathway diagnosis. Prog Retin Eye Res. 2001;20(4):531-61.

74. Bayer AU, Erb C. Short wavelength automated perimetry, frequency doubling technology perimetry, and pattern electroretinography for prediction of progressive glaucomatous standard visual field defects. Ophthalmology. 2002;109(5):1009-17.
75. Bach M, Hoffmann MB. Update on the pattern electroretinogram in glaucoma. Optom Vis Sci. 2008;85(6):386-95.

76. Ventura LM, Sorokac N, De Los Santos R, et al. The relationship between retinal ganglion cell function and retinal nerve fiber thickness in early glaucoma. Invest Ophthalmol Vis Sci. 2006;47(9):3904-11.

77. Schlottmann PG, De Cilla S, Greenfield DS, et al. Relationship between visual field sensitivity and retinal nerve fiber layer thickness as measured by scanning laser polarimetry. Invest Ophthalmol Vis Sci. 2004;45(6):1823-9.

78. Tafreshi A, Racette L, Weinreb RN, et al. Pattern electroretinogram and psychophysical tests of visual function for discriminating between healthy and glaucoma eyes. Am J Ophthalmol. 2010;149(3):488-95.

79. Bode SFN, Jehle T, Bach M. Pattern electroretinogram in glaucoma suspects: new findings from longitudinal study. Invest Ophthalmol Vis Sci. 2011;52(7):4300-6.

80. Forte R, Ambrosio L, Bonavolontà $\mathrm{P}$, et al. Pattern electroretinogram optimized for glaucoma screening (PERGLA) and retinal nerve fiber thickness in suspected glaucoma and ocular hypertension. Doc Ophthalmol. 2010;120(2):187-92.

81. Bowd C, Vizzeri G, Tafreshi A, et al. Diagnostic accuracy of pattern electroretinogram optimized for glaucoma detection. Ophthalmology. 2009;116(3):437-43.

82. Sehi M, Pinzon-Plazas M, Feuer WJ, et al. Relationship between pattern electroretinogram, standard automated perimetry, and optic nerve structural assessments. J Glaucoma. 2009;18(8): 608-17.

83. Yang A, Swanson WH. A new pattern electroretinogram paradigm evaluated in terms of user friendliness and agreement with perimetry. Ophthalmology. 2007;114(4):671-9.

84. Viswanathan S, Frishman LJ, Robson JG, et al. The photopic negative response of the flash electroretinogram in primary open angle glaucoma. Invest Ophthalmol Vis Sci. 2001;42(2):514-22.

85. Colotto A, Falsini B, Salgarello T, et al. Photopic negative response of the human ERG: losses associated with glaucomatous damage. Invest Ophthalmol Vis Sci. 2000;41(8):2205-11.

86. Machida S, Tamada K, Oikawa T, et al. Comparison of photopic negative response of full-field and focal electroretinograms in detecting glaucomatous eyes. J Ophthalmol. 2011;2011. doi: 10.1155/2011/564131.

87. Machida S, Gotoh Y, Toba Y, et al. Correlation between photopic negative response and retinal nerve fiber layer thickness and optic disc topography in glaucomatous eyes. Invest Ophthalmol Vis Sci. 2008;49(5):2201-7.

88. Machida S, Tamada K, Oikawa T, et al. Sensitivity and specificity of photopic negative response of focal electoretinogram to detect glaucomatous eyes. Br J Ophthalmol. 2010;94(2):202-8.

89. Hood DC, Greenstein VC. Multifocal VEP and ganglion cell damage: applications and limitations for the study of glaucoma. Prog Retin Eye Res. 2003;22(2):201-51.

90. De Moraes CG, Liebmann JM, Ritch R, et al. Understanding disparities among diagnostic technologies in glaucoma. Arch Ophthalmol. 2012;130(7):833-40.

91. Fortune B, Demirel S, Zhang X, et al. Comparing multipfocal VEP and standard automated perimetry in high-risk ocular hypertension and early glaucoma. Invest Ophthalmol Vis Sci. 2007;48(3):1173-80.

92. De Moraes CG, Liebmann JM, Ritch R, et al. Clinical use of multifocal visual-evoked potentials in glaucoma practice: a prospective study. Doc Ophthalmol. 2012;125(1):1-9. 